\title{
Early in vitro passages of breast cancer cells are differentially susceptible to retinoids and differentially express RAR $\beta$ isoforms
}

\author{
XINJIAN PENG ${ }^{1,2}$, ALBERT GREEN ${ }^{1}$, ANNE SHILKAITIS ${ }^{1}$, YONGHUA ZHU ${ }^{1}$, \\ LAURA BRATESCU $^{1}$ and KONSTANTIN CHRISTOV ${ }^{1}$
}

${ }^{1}$ Department of Surgery, University of Illinois at Chicago, Chicago, IL 60612; ${ }^{2}$ IIT Research Institute, Chicago, IL 60616, USA

Received February 25, 2011; Accepted April 18, 2011

DOI: 10.3892/ijo.2011.1070

\begin{abstract}
The effect of retinoids on breast cancer has been predominantly studied in vitro, on established cell lines, which in biology differ significantly from primary tumor cells. Little is known on whether early in vitro passages of breast cancer cells (EPBCCs) are differentially sensitive to retinoids and differentially express retinoid acid receptors (RARs) and retinoid X receptors (RXRs). We have previously identified a novel RAR $\beta$ isoform (RAR $\beta 5$ ) and hypothesized that it may serve as a potential target of retinoids in EPBCCs. Breast cancer cells isolated from primary tumors were cultured in vitro for 6-12 passages (EPBCCs) and their epithelial origin was confirmed by a cocktail of antibodies against cytokeratins. EPBCCs were treated for 4 days with $1.0 \mu \mathrm{M}$ of all-trans retinoic acid (atRA), 9-cis retinoic acid (9cRA) or 4-hydroxy-phenylretinamide (4-HPR) and their viability determined by MTT assay. Among nine EPBCCs consistently grown in vitro, three were resistant to the above retinoids, five were susceptible to atRA, four to 4-HPR and two to 9cRA, suggesting that patients with breast carcinomas may differentially respond to various retinoids. All EPBBCs differentially expressed RAR $\alpha, \operatorname{RAR} \gamma, \mathrm{RXR} \alpha, \mathrm{RXR} \beta$ proteins and RAR $\beta 5$ and RAR $\beta 2$ mRNAs. However, only one EPBCC (BCA-2) expressed RAR $\beta 5$ at mRNA and protein level and it was resistant to retinoids, both in vitro and in a xenograft tumor assay. RAR $\beta 5$ suppression by siRNA in BCA-2 cells increased their susceptibility to atRA. No correlation was
\end{abstract}

Correspondence to: Dr Konstantin Christov, Department of Surgical Oncology, University of Illinois at Chicago, 840 South Wood Street (M/C 820), Chicago, IL 60612, USA

E-mail: christov@uic.edu

Abbreviations: EPBCC, early passage of breast cancer; ER, estrogen receptor; atRA, all trans retinoic acid; 4-HPR, 4-hydroxyphenylretinamide; 9cRA, 9-cis retinoic acid; RARs, retinoic acid receptors; RARs $\alpha, \beta, \gamma$, retinoic acid receptors $\alpha, \beta, \gamma$; RXRs, retinoid $\mathrm{X}$ receptors; $\mathrm{RXR} \alpha, \beta, \gamma$, retinoid $\mathrm{X}$ receptors $\alpha, \beta, \gamma$

Key words: retinoids, breast cancer cells, retinoid receptors, RAR $\beta$, siRNA found between sensitivity of EPBCCs to the above retinoids and RAR $\beta 5$ and RAR $\beta 2$ mRNA expression. atRA reduced RAR $\beta$ expression in most EPBCCs suggesting that this retinoid receptor is most probably the prime target of retinoids in breast cancer. These data may have clinical implication in selecting patients with breast cancer that would benefit the most from clinical trials with retinoids.

\section{Introduction}

Most studies on breast cancer response to retinoids have been performed in vitro on established $\mathrm{ER}^{+}$and $\mathrm{ER}^{-}$cell lines, which significantly differ in biology from primary tumor cells $(1,2)$. Previously it was reported that $\mathrm{ER}^{+}$breast cancer cell lines are more sensitive to retinoids than ER ER $^{-}$ll lines $(3,4)$ and that retinoids may affect tumor cells by receptor-dependent and -independent mechanisms $(5,6)$. Retinoids are ligands of retinoic acid receptors (RARs $\alpha, \beta, \gamma)(7,8)$, whereas rexinoids preferentially affect retinoid X receptors (RXRs $\alpha, \beta, \gamma$ ). Both RARs and RXRs are expressed in normal mammary epithelial cells (MECs), whereas RAR $\beta$ and particularly its RAR $\beta 2$ isoform is lacking in most breast carcinomas, suggesting its potential tumor suppressor role $(9,11)$. The lack of RAR $\beta 2$ in breast cancer has been related to hypermethylation of the P2 promoter and its dimethylation has been associated with increased cell sensitivity to retinoids $(12,13)$. Transduction of RAR $\beta 2$ to MCF-7 cells lacking the receptor has been associated with decreased proliferation activity and increased sensitivity to retinoids (14). A truncated RAR $\beta$ ' isoform with a molecular mass of $40.6 \mathrm{kDa}$ has also been identified in some breast cancer cell lines, and its transduction to MCF-7 cells has been associated with increased cell growth and resistance to retinoids $(15,16)$.

We have recently identified a novel RAR $\beta$ isoform $(\beta 5)$, which has its own promoter, $\mathrm{P} 3$, different from the previously known P1 and P2 promoters. RAR $\beta 5$ was detected at the mRNA level in mammary premalignant MCF10AT cells and in some ER- breast cancer cell lines, all resistant to retinoids (17). The role of RAR $\beta 5$ on malignant properties of breast tumor cells and on their sensitivity to retinoids is currently under investigation in our laboratory. In addition to RAR $\beta 5$, short 5'-UTR RAR $\beta 2$ transcripts were also detected in breast cancer cell lines and these transcripts may also serve as potential targets of retinoids (18). In this study, we characterized 
the susceptibility of EPBCC isolated from primary tumors to atRA, 9cRA, and 4-HPR and found 3 of 9 (33\%) resistant to all three retinoids, whereas the other 6 more sensitive to atRA and 4-HPR than to 9cRA. All EPBCC differentially expressed RAR $\beta 5$ and RAR $\beta 2$ mRNA, as well as RARs $\alpha, \gamma$ and RXRs $\alpha, \beta$ with most significant decrease in $\operatorname{RAR} \alpha$, which appears the principal target of retinoids in breast cancer.

\section{Material and methods}

In vitro growth and establishment of EPBCC. Tumor cells isolated from primary and metastatic breast carcinomas have been continuously cultured in vitro for 6-12 passages. UIC IRB approval was obtained prior to conducting this study, and informed consents were obtained from patients undergoing surgery for breast cancer prior to culturing of tumor cells in vitro. When cells from minced tissue formed a single layer in culture flasks, cells were passaged and cultured in MEM supplemented with $100 \mu \mathrm{g} / \mathrm{ml}$ penicillin, $100 \mu \mathrm{g} / \mathrm{ml}$ streptomycin and 10\% FBS, $200 \mu \mathrm{M}$ L-glutamine and $100 \mu \mathrm{M}$ MEM non-essential amino acid. The epithelial origin of BCC was confirmed by immunocytochemistry ( $\mathrm{ICH})$ with a cocktail of antibodies against cytokeratins: 5, 8, 14 and 18 (data not shown). When the cells grown in culture reached $30-50 \%$ confluence, they were treated with retinoids or DMSO (solvent control). T47D cells, which serve as controls, were obtained from the American Cell Type Collection, Manassas, VA. The conditions for cell culturing are described in our recent publication (19).

Retinoids. atRA and 9cRA were purchased from Sigma, Inc., St. Louis, MO. 4-HPR was obtained from the repository of the National Cancer Institute (Bethesda, MD). Retinoids were dissolved in DMSO and added to the cell culture medium at $1.0 \mu \mathrm{M}$ every other day for 4 days.

MTT assay. Effect of retinoids on cell growth and viability was determined by MTT (3-(4, 5-dimethylthiazol-2-yl)-2,5diphenyl tetrasolium bromide) assay. Cells (1000 cells/well) were seeded in 96-well plates and cultured overnight. Then the cells were incubated with $1 \mu \mathrm{M}$ of different retinoids. After a 4-day treatment, $10 \mu \mathrm{l}$ MTT $(5 \mathrm{mg} / \mathrm{ml})$ was added to each well, mixed gently and incubated with the cells at $37^{\circ} \mathrm{C}$ for 1-3 h (19).

$R T$-PCR. Total RNA extraction and RT reaction were performed as described previously (17-19). Two RT reactions for each sample were pooled and diluted with an equal amount of DNase/RNase free water. For RT, a final volume of $20 \mu \mathrm{l}$ with $2 \mu \mathrm{g}$ total RNA and 100 units of MuLV reverse transcriptase (Invitrogen) at $42^{\circ} \mathrm{C}$ for $50 \mathrm{~min}$ was used. PCR was performed with $1 \mu \mathrm{l}$ RT product using PCR Supermix (Invitrogen). PCR primer pairs are RAR $\beta 2$ (475FP, GACTGTATGGATGT TCTGTCAG; 730RP, ATTTGTCCTGGCAGACGAAGCA), RAR 35 (14FP, CTGGAAGGTCGTACACAGTGA; 343RP, GGACATTCCCACTTCAAAGC). $\beta$-actin (FP, GTCACCA ACTGGGACGACA; RP, TGGCCATCTCTTGCTCGAA) was used as an internal control. Real-time PCR was performed with $1 \mu \mathrm{l}$ RT product using 7900HT Sequence Detection System (ABI, Applied Biosystem) and ABI 2X SYBR Green PCR Master Mix (ABI\# 4309155) according to ABI's
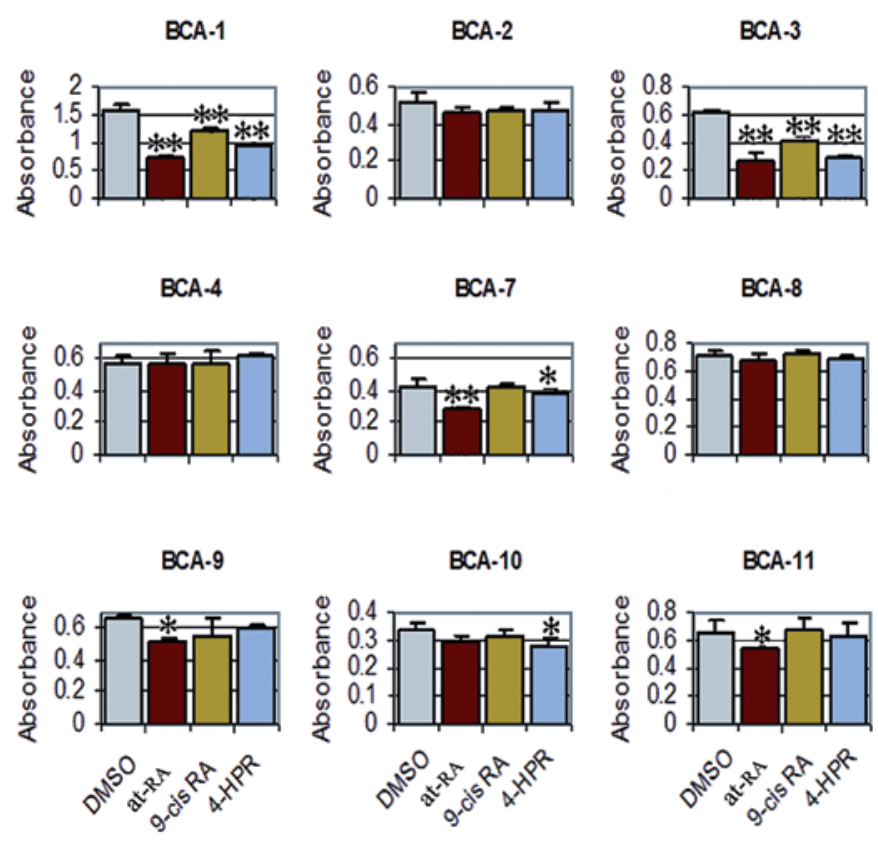

Figure 1. Effects of atRA, 9cRA and 4-HPR on the growth/viability of EPBCC. Cells were treated for 4 days with $1.0 \mu \mathrm{M}$ of the above retinoids and subjected to MTT assay. Data are expressed as the mean absorbance \pm SD of 8 wells. All data are representative of three independent experiments. " $\mathrm{p}<0.05$ compared to control; ${ }^{* *} \mathrm{p}<0.01$ compared to control. Note that atRA was the most efficacious of the 3 retinoids examined.

recommended guidelines. Primer pairs for real-time PCR were RAR 32 (584FP, GATTGACCCAAACCGAATGGCA GCA; 730RP) and RAR $\beta 5$ (15FP, GGAAGGTCGTACAC AGTGAATTTCTCTGAG; RARß2-730RP); real-time PCR data were analyzed using a software package (ABI Prism SDS2.1) provided with the instrumentation system.

Inhibition of RAR 35 expression by siRNA. Two siRNAs were designed to target the sequences 5'-AAA ATT CTG GAA GGT CGT ACA-3' and 5'-AAT TCT GGA AGG TCG TAC ACA-3', both of which are present in exon-6, a region unique to RAR $\beta 5$. To suppress RAR $\beta 5$ expression by siRNA, Ambion Inc. (Houston, TX) made the constructs and we transfected siRNA to BCA-2 cells. The efficacy of transfection was very high $(>90 \%)$. We first determined the concentration of siPORT NeoFX transfection agent that was not toxic by using an MTT assay. Then we employed 3 concentrations of siPORT NeoFX, $0.3,0.6$ and $1.0 \mu \mathrm{l}$, and siRNA at $0.1,0.5$ and $1.0 \mathrm{nM}$. siRNA transfected ( 0.1 and $1.0 \mathrm{nM})$, vector-transfected, and control cells were treated with atRA, $1.0 \mu \mathrm{M}$ for 2 days, and the effect of siRNA on RAR $\beta 5$ expression was evaluated by Western blot. Another set of siRNA transfected, dsRNA transfected and control cells were treated with atRA, $1.0 \mu \mathrm{M}$ for 3 days and their viability estimated by MTT assay (20).

Western blot and immunocytochemistry (ICH). Western blotting was used to detect RAR $\beta 5, \operatorname{RAR} \beta 2, \operatorname{RAR} \alpha, \operatorname{RAR} \gamma$, $\mathrm{RXR} \alpha$ and $\mathrm{RXR} \beta$ expression (RXR $\gamma$ was not examined). Two antibodies were used to detect RAR $\beta$ isoforms, one recognizing amino acids 430-447 in the C-terminus of RAR $\beta 2$ (sc-552, Santa Cruz Biotechnology, Inc., Santa Cruz, CA) and the other one recognizing amino acids 407-423 in the C-terminus 


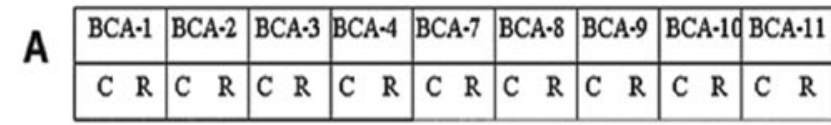
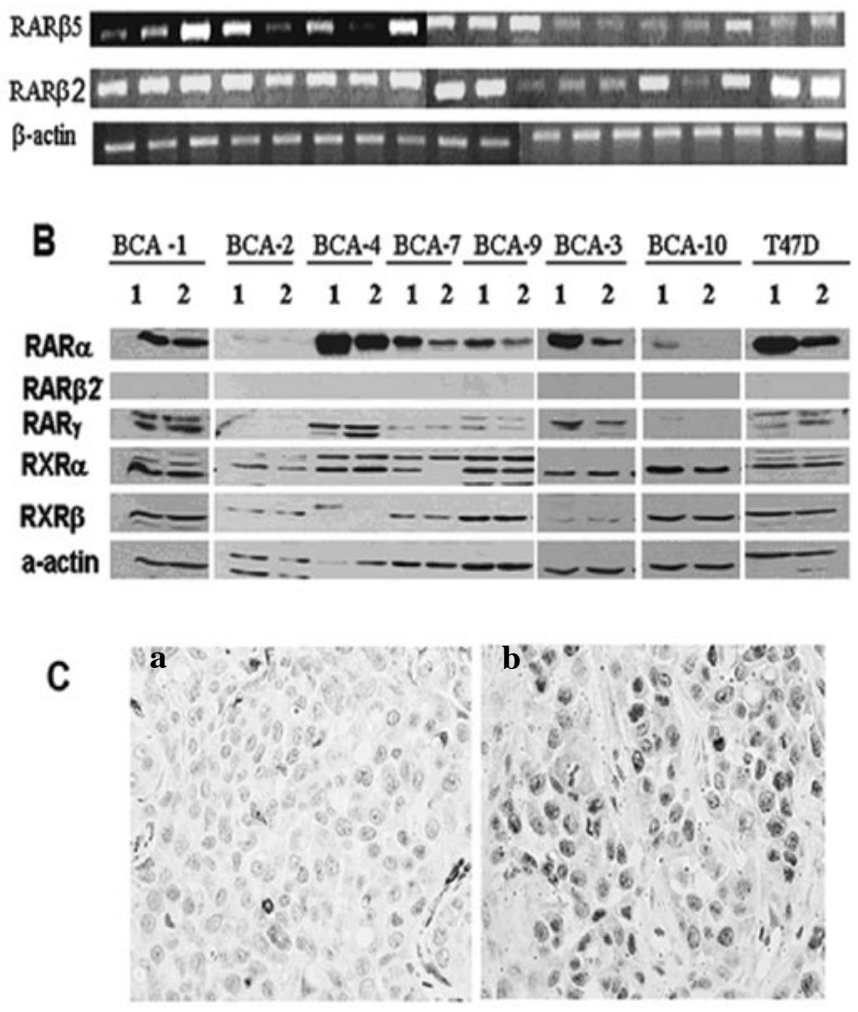

Figure 2. RAR $\beta 5$ and RAR $\beta 2$ expression in EPBCC. (A) RT-PCR analysis of RAR $\beta 5$ and RAR $\beta 2$ mRNA. The cells were treated with $1.0 \mu \mathrm{M}$ atRA (R) for 4 days. Control cells (C) were treated with DMSO (solvent). (B) Differential expression of RAR $\alpha, \operatorname{RAR} \beta 2, \mathrm{RAR} \gamma, \mathrm{RXR} \alpha$, and RXR $\beta$ in EPBCC, Western blot data. The total protein was isolated and processed for Western blotting. T47D cells were used as negative control of both RAR $\beta 5$ and RAR $\beta 2$. RAR $\beta 2$ was not identified at the protein level in EPBCC. Note, that atRA decreased RAR $\alpha$ in most EPBCC. (C) RAR 35 expression in BCA-2 tumor xenografts, as determined by immunocytochemistry with sc-552 antibody and $\mathrm{ABC}$ kit. (a) A negative control without antibody treatment and (b) parallel slide treated with the above antibody. RAR $\beta 5$ was preferentially expressed in the nucleus of tumor cells. The slides are counter-stained with hematoxylin, $x 400$.

of RAR $\beta 2$ (Geneka Inc., Montreal, Quebec). Antibodies for RAR $\alpha, \operatorname{RAR} \gamma, \operatorname{RXR} \alpha$, and RXR $\beta$ were also purchased from Santa Cruz Biotechnology. For ICH, paraffin sections from tumor xenografts of BCA-2 cells that express RAR $\beta 5$ protein and BCA- 1 cells that do not express RAR $\beta 5$ were deparaffinized and treated with $0.01 \mathrm{M}$ citric buffer in a pressure cooker for 10 min. Next, the slides were blocked with mouse serum to eliminate non-specific staining and incubated with antibody for $2 \mathrm{~h}$ atroom temperature. ABC elitekitand 3,3'-diaminobenzidinetetrahydrochloride (DAB) were used to identify cells that expressed RAR $\beta 5$. Parallel sections were not treated with antibody and served as negative controls.

Nude mouse xenograft tumor assay. For this study, BCA-1 cells (sensitive in vitro to atRA) and BCA-2 cells (resistant to atRA) were mixed with Matrigel $\left(10^{6}\right.$ cells in $0.1 \mathrm{ml}$ Matrigel $)$ and injected in the mammary fat pad of 4-5 week old nude mice. The occurrence of tumor nodules was monitored weekly. Animals with palpable tumors $(2-3 \mathrm{~mm})$ were treated by gavage with atRA, $10.0 \mathrm{mg} / \mathrm{kg}$ body wt for 4 weeks. Control animals received a placebo (sesame oil). After sacrifice of the animals by $\mathrm{CO}_{2}$ asphyxiation, tumor nodules were removed, fixed in buffered, $\mathrm{pH} 7.4$ formalin and embedded in paraffin. Samples from tumors were also frozen in liquid nitrogen for RNA and protein analysis. Mice were cared for in accordance with the procedures outlined in the NIH Guide for the Care and Use of Laboratory Animals.

Statistical analysis. Statistical analysis of cell growth determined by MTT assay and the volume of control and atRA-treated tumor xenografts are given in the last paragraph of the Material and methods section. Three independent tests for cell viability (MTT assay) were performed and the mean value \pm standard deviation (SD) are presented. The difference with control (placebo treated) cells or treated tumors are calculated by two-sided Student's t-test. Differences in p-values of $<0.05$ are considered significant.

\section{Results}

EPBCC differ in their susceptibility to retinoids. We examined 9 EPBCC (passage 6-12) out of 29 cell lines developed in our laboratory, to determine their susceptibility to retinoids. These EPBCC grew consistently in vitro and when transplanted in nude mice developed tumor xenografts, thus confirming their malignant properties. In a preliminary study, we found that it takes 3-4 days for retinoids at pharmacological doses to suppress the growth of breast normal and cancer cell lines (20). Tumor cells were treated for 4 days with $1.0 \mu \mathrm{M}$ of atRA, 9cRA or 4-HPR, and MTT data for cell viability were compared with control (placebo-DMSO) cells (Fig. 1). Of nine EPBCC, five were susceptible to atRA (BCA-1, BCA-3, BCA-7, BCA-9 and BCA-11), four to 4-HPR (BCA-1, BCA-3, BCA-7 and BCA-10), and two to 9cRA (BCA-1 and BCA-3), indicating that EPBCC are differentially sensitive to retinoids. Interestingly, BCA-2, BCA-4 and BCA- 8 were resistant to all three retinoids, suggesting that breast carcinomas could be divided into two groups, susceptible and resistant to retinoids.

$R A R \beta 5$ and $R A R \beta 2$ isoforms are differentially expressed in $E P B C C$ and differentially respond to retinoids. Both RAR $\beta$ isoforms were examined at mRNA and protein levels. As shown (Fig. 2A) RAR $\beta 5$ and RAR $\beta 2$ mRNA are differentially expressed in all EPBCC and differentially respond to atRA. For instance, the high level of RAR $\beta 5$ mRNA expression in BCA-2 and BCA- 8 cells correlated with their resistance to all three retinoids, whereas the low level in BCA-1, BCA-3, BCA-9, BCA-10, and BCA-11 cells correlated with their sensitivity to atRA. However, RAR $\beta 5$ mRNA was barely detected in BCA-4 cells, but they were resistant to retinoids. To assess the effect of atRA on RAR $\beta 5$ and RAR $\beta 2$ expression, cells were treated with $1.0 \mu \mathrm{M}$ atRA for 1,2 , or 4 days and mRNA and proteins levels examined (Fig. 2A). It is evident that atRA preferentially affected RAR $\beta 5$ mRNA expression. However, when the data for alterations in both RAR $\beta$ isoforms were compared with cell sensitivity to retinoids (Fig. 1), no correlation was found. At the protein level, EPBCC did not express RAR $\beta 2$ (Fig. 2B), only one (BCA-2) expressed RAR $\beta 5$ isoform, and the cells were resistant to retinoids both 
A
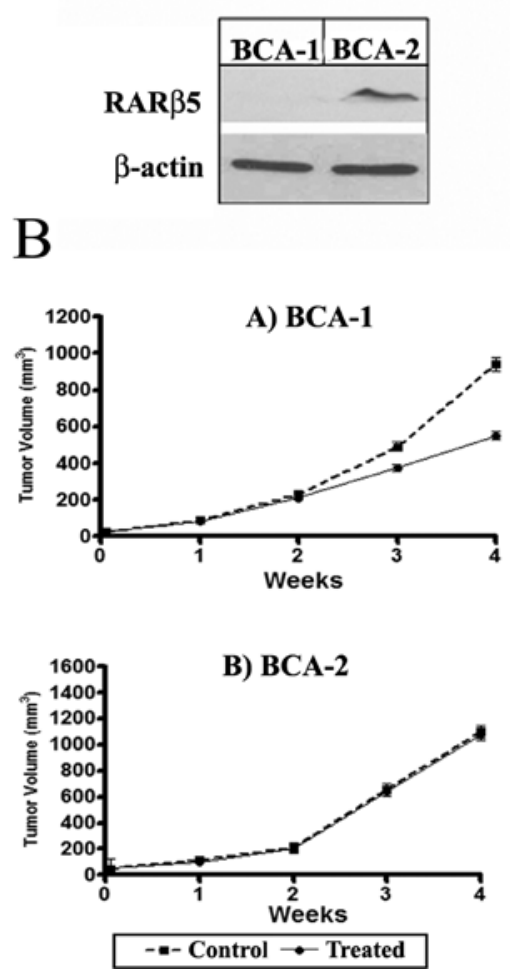

Figure 3. RAR $\beta 5$ protein expression and cell sensitivity to atRA. (A) Western blot data. RAR $\beta 5$ protein was detected by sc-552 polyclonal antibody in resistant BCA-2, but not in sensitive to atRA BCA-1 cells. (B) The effect of atRA on the growth of BCA-1 (a) and BCA-2 (b) tumor xenografts. Nude mice were injected with $10^{6}$ cells mixed with matrigel in fad pets of abdominal mammary gland. When palpable tumors occurred animals were separated into control (6 mice) and treated with atRA (6 mice) groups. atRA was given by gavage at $40 \mathrm{mg} / \mathrm{kg}$ body weight 6 days a week for 4 weeks. Sesame oil was used as placebo. Tumor volume was measured by caliper once a week. Note, the decrease in BCA-1 tumor xenografts after treatment with atRA, as compared to placebo treated animals. The same dose of atRA was not efficacious in animals with BCA-2 xenografts.

in vitro and in a xenograft tumor assay (Fig. 3A-C). $\mathrm{ICH}$ detected RAR $\beta 5$ protein only in BCA-2 cells, and it was mostly localized in the nucleus (Fig. 3C). Interestingly, in BCA- 2 cells, RAR $\beta 2$, RAR $\alpha$ and RAR $\gamma$ proteins were also not detectable and their expression was not affected by atRA, suggesting that in a subset of breast carcinoma a disruption of all RARs may occur. The remaining EPBCC differentially expressed RAR $\alpha, \mathrm{RAR} \gamma, \mathrm{RXR} \alpha$ and RXR $\beta$ protein (RXRg was not examined) and differentially responded to atRA treatment (Fig. 2B). The most consistent decrease was found in $\mathrm{RAR} \alpha$ protein, which appears the principal target of retinoids in breast cancer cells.

Knock down of RARB5 by siRNA increased cell sensitivity to $a t R A$. To further characterize the potential role of RAR $\beta 5$ expression on the sensitivity of breast cancer cells to retinoids, we knocked down RAR $\beta 5$ expression by siRNA in BCA-2 cells (Fig. 4A). Ambion Inc. was provided with the RAR $\beta 5$ sequences of sense- and anti-sense strand siRNA. They made the constructs and we transfected the corresponding siRNA to BCA-2 cells. Vector-transfected (VT), dsRNA, and siRNA

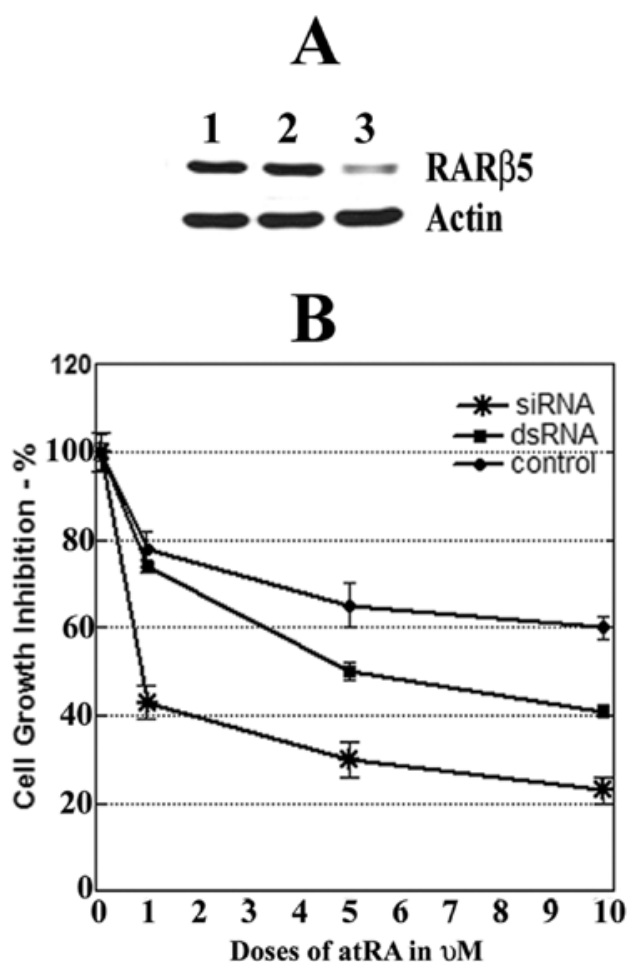

Figure 4. (A) Effects of siRNA on RAR $\beta 5$ expression in BCA-2 cells. Cells were treated for 2 days with $1.0 \mu \mathrm{M}$ atRA: In the top line are presented cells transfected with VT (1); dsRNA, cells treated with double stranded RNA (2); siRNA, cells transfected with siRNA by employing $1.0 \mathrm{nM}$ of siRNA (3). The expression level of RAR $\beta 5$ was detected by Western blotting. $\beta$-actin was used as a loading control. (B) atRA induced cell growth inhibition in siRNA-transfected cells. atRA at $1.0,5.0$ and $10 \mu \mathrm{M}$ was added to the culture medium for 4 days and cell viability/growth was detected by MTT assay (see Material and methods). Vector-transfected cells appear also more sensitive to retinoids than control cells.

$(0.1$ and $1.0 \mathrm{nM})$ transfected cells were treated with $1.0 \mu \mathrm{M}$ atRA for 2 days and the effects of siRNA on RAR $\beta 5$ expression was evaluated by Western blotting. Fig. 4A shows that siRNA significantly suppressed RAR $\beta 5$ expression. However, the cells transfected with an empty vector also demonstrated limited sensitivity to atRA, suggesting that the empty vector may affect cellular targets of atRA not necessarily associated with RAR $\beta$ isoforms. In a second set of experiments, RAR $\beta 5$ and vector transfected cells were treated with atRA, $1.0 \mu \mathrm{M}$ for 3 days, in 96-well plates and their viability estimated by MTT assay (Fig. 4B). The increase in atRA dose from 1.0 to 5.0 and $10 \mu \mathrm{M}$ progressively suppressed cell growth, indicating that inhibition of RAR $\beta 5$ expression by siRNA increases tumor cell sensitivity to retinoids.

RAR $\beta 5$ expression in BCA- 2 cells correlates with their in vivo resistance to atRA. To determine whether the resistance of BCA-2 cells to retinoids in vitro may persist in vivo, $10^{6}$ cells were mixed in Matrigel and transplanted in mammary fat pads of nude mice (6 mice per group). BCA-1 cells were used in a parallel experiment as a cell line sensitive to retinoids. When palpable tumors occurred (3-4 $\mathrm{mm}$ ) the animals were randomized in control and atRA-treated groups. atRA was given by gavage at $40.0 \mathrm{mg} / \mathrm{kg}$ body weight for 4 weeks, 6 days a weeks. In a preliminary study, we found that atRA at 

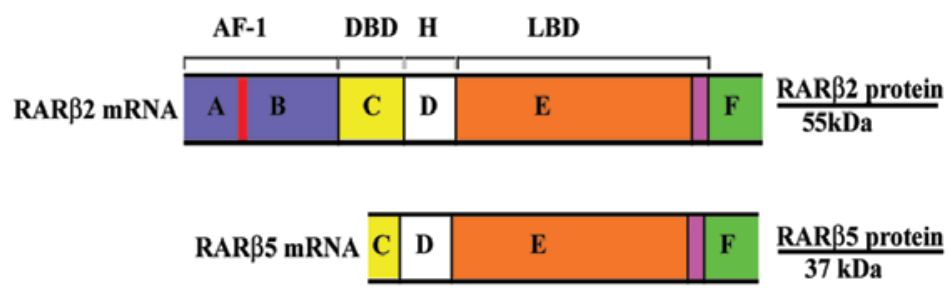

Figure 5. Differential structural organization of RAR $\beta 2$ and RAR $\beta 5$ receptor isoforms in breast cancer cells. Protein domains (A-F) of RAR $\beta$ isoforms are depicted: N-terminal region, which comprises A + B of AF-1 and is expressed in RAR $\beta 2$ but lacking in RAR $\beta 5$; DNA binding domain (DBD) C, lacks one of the two zinc fingers in RAR $\beta 5$; $\mathrm{H}$, hinge; and ligand binding domain (LBD), which comprises E and part of F of the receptor protein. Note the difference in molecular mass between RAR $\beta 2(55 \mathrm{kDa})$ and RAR $\beta 5(37 \mathrm{kDa})$ isoforms.

the above dose was not toxic and did not affect the body weight of the animals. As shown in Fig. 3B, atRA reduced the growth of sensitive BCA-1, but not that of resistant BCA-2 xenografts that expressed RAR $\beta 5$.

\section{Discussion}

The main objective of this study was to assess the feasibility of EPBCC as a model system in testing the antitumor efficacy of retinoids and to determine the role of RAR $\beta 5$ and RAR $\beta 2$ isoforms as potential targets of retinoids. EPBCC appear closer in biology, ER status and genetic background to primary tumor cells than to established breast cancer cell lines (21). However, it is not easy to establish EPBCC consistently growing in vitro. It takes at least 3-5 passages of primary breast cancer cells to clear up the non-epithelial cell populations from the culture.

We confirmed the epithelial origin of EPBCC by treating cells with a cocktail of antibodies against cytokeratins (data not shown). atRA, 9cRA, and 4-HPR were employed because they differentially affect RARs and RXRs and in previous studies have shown efficacy in breast cancer preclinical and clinical studies $(7,22,23)$. atRA is a ligand of RARs $\alpha, \beta, \gamma$, 9cRA activates both, RARs and RXRs, and 4-HPR is a weak ligand of RAR $\gamma$ or does not need retinoid receptors to exert its antitumor effect (24).

We found that EPBCC differed in their sensitivity to the above retinoids; most (5 of 9) were sensitive to atRA and 4-HPR (4 of 9) but only two to 9cRA, suggesting that breast carcinomas are differentially susceptible to various retinoids. Surprisingly, 33\% (3 of 9) of EPBC were resistant to all three retinoids, suggesting that patients with these tumors may not benefit from clinical trials with retinoids. Several clinical studies with retinoids support our data. For instance, in a phase II breast cancer clinical trial with atRA, response has been observed in individual patients only (22). In a breast cancer prevention trial with 4-HPR that continued for more than 8 years, a $30 \%$ reduction in cancer development has been found in pre-menopausal women, data further supporting our results (23). Preclinical and clinical studies have shown that atRA and 9cRA are relatively toxic; therefore their clinical implication is limited.

Recently, rexinoids (LGD1069, LG10068, UAB30), which are ligands of RXRs, have shown low toxicity and promising efficacy in inhibiting mammary carcinogenesis in animal models $(8,25,26)$. Some of the above rexinoids are currently employed in clinical trials for the treatment of breast and other types of cancer (27). Most studies on breast cancer cell lines, including ours, have shown that retinoids can suppress cell and tumor growth by inhibiting cell proliferation, inducing differentiation, cellular senescence and/or apoptosis $(28,29)$. In mediating these cellular events, retinoids can modulate the expression of RARs and RXRs or employ receptor-independent mechanisms (30). As shown in Fig. 2, all EPBCC differentially expressed RARs, $\alpha, \gamma$ and RXRs, $\alpha, \beta$, but not RAR $\beta 2$ protein, supporting previous studies indicating that $R A R \beta 2$ is lacking in most breast cancers. Here, we provide data that in addition of RAR $\beta 2$, RAR $\beta 5$ mRNA was identified in all BCC. RAR $\beta 5$ differs from RAR $\beta 2$ and RAR $\beta 4$ in exon organization (exon 5a) and in initiating point of translation (Fig. 5). Most importantly, RAR $\beta 5$ has a distinct promoter, $\mathrm{P} 3$, different from the previously known P1 and P2 promoters of the RAR $\beta 1$, RAR $\beta 2$ and RAR $\beta 4$ isoforms $(31,32)$. Recently it was shown that RAR $\beta 2$ expression in stroma cells may promote not suppress mammary carcinogenesis bringing a new level of complexity in assessing the efficacy of retinoids to suppress mammary carcinogenesis (33).

Since, as we have previously shown, RAR $\beta 5$ is predominantly expressed in ER-negative breast cancer cell lines most of which are resistant to retinoids $(18,19)$, we hypothesized that this isoform may predict the resistance/sensitivity of EPBCC to retinoids. The data generated in this study do not support this hypothesis. The lack of correlation between RAR $\beta 5$ mRNA expression and the sensitivity of EPBCC to atRA suggests that other members of RARs and/or RXRs might be responsible for cell sensitivity to retinoids. RAR $\beta 2$ should be also excluded, because its mRNA values do not correlate with cell sensitivity to retinoids. The data from Western blotting suggests that primary target of atRA in EPBCC is most probably RAR $\alpha$, the expression of which is suppressed by atRA in all but one EPBCC. Previous studies on MCF-cells support our data that RAR $\alpha$ is the primer target of atRA (6). The decrease of RAR $\alpha$ expression may affect RAR $\beta 2$ transcription and further sensitivity of tumor cells to retinoids $(7,34,35)$. Interestingly, BCA-2 cells constitutively expressed RAR $\beta 5$, but not RAR $\alpha$, RAR $\beta 2$, or RAR $\gamma$ proteins, suggesting significant post-translational defects in RAR signaling that correlated with cell resistance to retinoids both in vitro and in vivo. To further prove the potential role of RAR $\beta 5$ in the resistance of BCA-2 cells to retinoids, we knocked down RAR $\beta 5$ by siRNA; this was associated with increased cell susceptibility to atRA, suggesting that at least in 
a subset of breast carcinomas, RAR $\beta 5$ may serve as a potential biomarker of cell resistance to atRA and possibly to other retinoids. The response of breast cancer cells to retinoids is a complex phenomenon involving not only receptor-dependent but also receptor-independent mechanisms. In addition, there are co-activators and co-repressors of the RAR isoforms that might also affect the sensitivity of cells to retinoids $(7,11)$. For instance, 4-HPR, which is known not to affect retinoid receptors or may weakly activate $\operatorname{RAR} \gamma(24)$, suppressed the growth of 4 of 9 EPBCC.

In conclusion, in this study we found that $\sim 30 \%$ of EPBCC are resistant in vitro to retinoids, whereas the remaining are differentially sensitive to atRA, 9cRA, and 4-HPR. The response of EPBCC to retinoids is a complex phenomenon and do not depend on the expression levels of RAR $\beta 5$ and RAR $\beta 2$ receptors only. The data may help in selecting patients that benefit the most from clinical trials with retinoids.

\section{Acknowledgements}

This work was supported by Suzan G. Komen KG100509 grant (K.C.) and Illinois Department of Public Health Penny Stevens Breast and Cervical Cancer Research Fund (X.P.). We thank Dr Rajeshwari Mehta for her support and encouragement.

\section{References}

1. Roman SD, Clarke CL, Hall RE, Alexander IE and Sutherland RL: Expression and regulation of retinoic acid receptors in human breast cancer cells. Cancer Res 52: 2236-2242, 1992.

2. Liu Y, Lee MO, Wang HG, Li Y, Hashimoto Y, Klaus M, Reed JC, et al: Retinoic acid receptor beta mediates the growthinhibitory effect of retinoic acid by promoting apoptosis in human breast cancer cells. Mol Cell Biol 16: 1138-1149, 1996.

3. Ueda H, Ono M, Hagino Y and Kuwano M: Isolation of retinoic acid-resistant clones from human breast cancer cell line MCF-7 with altered activity of cellular retinoic acid-binding protein. Cancer Res 45: 3332-3338, 1985.

4. Gauthier ML, Berman HK, Miller C, et al: Abrogated response to cellular stress identifies DCIS associated with subsequent tumor events and defines basal-like breast tumors. Cancer Cell 12: 479-491, 2007.

5. Song S, Guan B, Men T, Hoque A, Lotan R and Xu XC: Antitumor effect of retinoic acid receptor-beta2 associated with suppression of cyclooxygenase-2. Cancer Prev Res 2: 274-280, 2009.

6. Tanaka T, Rodriguez de la Concepcion ML and De Luca LM: Involvement of all-trans-retinoic acid in the breakdown of retinoic acid receptors alpha and gamma through proteasomes in MCF-7 human breast cancer cells. Biochem Pharmacol 6: 1347-1355, 2001.

7. Lotan R: Retinoids in cancer chemoprevention. FABS J 10: 1031-1039, 1996.

8. Liby KT, Yore MM and Sporn MB: Triterpenoids and retinoids as multifunctional agents for the prevention and treatment of cancer. Nat Rev Cancer 7: 357-369, 2007

9. Xu XC, Sneige N, Liu X, et al: Progressive decrease in nuclear retinoic acid receptor beta messenger RNA level during breast carcinogenesis. Cancer Res 57: 4992-4996, 1997.

10. Widschwendter M, Berger J, Daxenbichler G, Muller-Holzner E, Widschwendter A, Mayr A, Marth C and Zeimet AG: Loss of retinoic acid receptor beta expression in breast cancer and morphologically normal adjacent tissue but not in the normal breast tissue distant from the cancer. Cancer Res 57: 4158-4161, 1997.

11. Yang Q, Sakurai T and Kakudo K: Retinoid, retinoic acid receptor beta and breast cancer. Breast Cancer Res Treat 76: 167-173, 2002.

12. Widschwendter M, Berger J, Hermann M, et al: Methylation and silencing of the retinoic acid receptor-beta 2 gene in breast cancer. J Natl Cancer Inst 92: 826-832, 2000.
13. Sirchia SM, Ferguson AT, Sironi E, Subramanyan S, Orlandi R, Sukumar S and Sacchi N: Evidence of epigenetic changes affecting the chromatin state of the retinoic acid receptor beta2 promoter in breast cancer cells. Oncogene 19: 1556-1563, 2000.

14. Wallden B, Emond M, Swift ME, Disis ML and Swisshelm K: Antimetastatic gene expression profiles mediated by retinoic acid receptor beta 2 in MDA-MB-435 breast cancer cells. BMC Cancer 5: 140-148, 2005.

15. Chen LI, Sommer KM and Swisshelm K: Downstream codons in the retinoic acid receptor beta- 2 and beta- 4 mRNAs initiate translation of a protein isoform that disrupts retinoid-activated transcription. J Biol Chem 277: 35411-35421, 2002.

16. Swift ME, Wallden B, Wayner EA and Swisshelm K: Truncated RAR beta isoform enhances proliferation and retinoid resistance. J Cell Physiol 209: 718-725, 2006.

17. Peng X, Maruo T, Cao Y, Punj V, Mehta R, Das Gupta TK and Christov K: A novel RARbeta isoform directed by a distinct promoter $\mathrm{P} 3$ and mediated by retinoic acid in breast cancer cells. Cancer Res 64: 8911-8918, 2004.

18. Peng X, Mehta RG, Tonetti DA and Christov K: Identification of novel RARbeta2 transcript variants with short 5'-UTRs in normal and cancerous breast epithelial cells. Oncogene 24: 1296-1301, 2005.

19. Peng X, Yun D and Christov K: Breast cancer progression in MCF10A series of cell lines is associated with alterations in retinoic acid and retinoid $X$ receptors and with differential response to retinoids. Int J Oncol 25: 961-971, 2004.

20. Peng X, Wood S, Bratescu L, Shilkaitis A and Christov K: Retinoids suppress premalignant MCF10AT but not malignant MCF10CAla breast epithelial cells in vivo. Role of retinoic acid receptor beta 2 expression. Cancer Lett 222: 153-163, 2005.

21. Hass R and Bertram C: Characterization of human breast cancer epithelial cells (HBCEC) derived from long term cultured biopsies. J Exp Clin Cancer Res 28: 2-12, 2009.

22. Sutton LM, Warmuth MA, Petros WP and Winer EP: Pharmacokinetics and clinical impact of all-trans retinoic acid in metastatic breast cancer: a phase II trial. Cancer Chemother Pharmacol 40: 335-341, 1997.

23. Veronesi U, De Palo G, Marubini E, Costa A, Formelli F, Mariani L, Decensi A, et al: Randomized trial of fenretinide to prevent second breast malignancy in women with early breast cancer. J Natl Cancer Inst 91: 1847-1856, 1999.

24. Fanjul AN, Bouterfa H, Dawson M and Pfahl M: Potential role for retinoic acid receptor-gamma in the inhibition of breast cancer cells by selective retinoids and interferons. Cancer Res 56: 1571-1577, 1996.

25. Bischoff ED, Gottardis MM, Moon TE, Heyman RA and Lamph WW: Beyond tamoxifen: the retinoid X receptorselective ligand LGD1069 (TARGRETIN) causes complete regression of mammary carcinoma. Cancer Res 58: 479-484, 1998.

26. Grubbs CJ, Lubet RA, Atigadda VR, et al: Efficacy of new retinoids in the prevention of mammary cancers and correlations with short-term biomarkers. Carcinogenesis 27: 1232-1239, 2006.

27. Esteva FJ, Glaspy J, Baidas S, et al: Multicenter phase II study of oral bexarotene for patients with metastatic breast cancer. J Clin Oncol 21: 999-1006, 2003.

28. Christov K: The novel RARbeta isoform (beta5) is a potential target of retinoids in breast cancer. Curr Cancer Drug Targets 9: 142-147, 2009.

29. Wu K, Kim HT, Rodriquez JL, et al: Suppression of mammary tumorigenesis in transgenic mice by the RXR-selective retinoid, LGD1069. Cancer Epidemiol Biomarkers Prev 1: 467-474, 2002.

30. Chen Y, Dokmanovic M, Stein WD, Ardecky RJ and Roninson IB: Agonist and antagonist of retinoic acid receptors cause similar changes in gene expression and induce senescencelike growth arrest in MCF-7 breast carcinoma cells. Cancer Res 66: 8749-8761, 2006.

31. Dokmanovic M, Chang BD, Fang J and Roninson IB: Retinoidinduced growth arrest of breast carcinoma cells involves co-activation of multiple growth-inhibitory genes. Cancer Biol Ther 1: 24-27, 2002.

32. Seewaldt VL, Johnson BS, Parker MB, Collins SJ and Swisshelm K: Expression of retinoic acid receptor beta mediates retinoic acid-induced growth arrest and apoptosis in breast cancer cells. Cell Growth Differ 6: 1077-1088, 1995. 
33. Liu X, Nugoli $\mathrm{M}$, Laferrière $\mathrm{J}$, et al: Stromal retinoic acid receptor (beta) promotes mammary gland tumorigenesis. Proc Natl Acad Sci USA 108: 774-779, 2011

34. Lu M, Mira-y-Lopez R, Nakajo S, Nakaya K and Jing Y: Expression of estrogen receptor alpha, retinoic acid receptor alpha and cellular retinoic acid binding protein II genes is coordinately regulated in human breast cancer cells. Oncogene 24: 4362-4369, 2005.

35. Ren M, Pozzi S, Bistulfi G, Somenzi G, Rossetti S and Sacchi N: Impaired retinoic acid (RA) signal leads to RARbeta2 epigenetic silencing and RA resistance. Mol Cell Biol 25: 10591-10603, 2005. 